But if you are hungry for new methods, what does this issue have to offer? In terms of improvements to existing high-throughput screens, two papers propose complementary enhancements to existing approaches. On page 1013, the group of Angela Bauch and Giulio Superti-Furga present new tags for tandem affinity purification (TAP), the workhorse of two recent genome-wide interactome studies in yeast. The new tags provide higher yields and better specificity, opening the door to TAP-tag purification and mass spectrometry analysis in mammalian cells, where so far the procedure has performed poorly.

Although this type of approach has fantastic scalability (virtually all proteins can be tagged), adding tags to proteins may affect their behavior-a problem that interactome studies must address by using different tags at different positions in proteins. More fundamentally, the requirement for protein overexpression can be problematic, even though it does improve sensitivity. In contrast, focusing on endogenous proteins has typically been hampered by the need for good antibodies and the lack of specificity. In the report on page 981, Matthias Selbach and Matthias Mann address the specificity problem by combining RNAi-based 'negative selection' with their previously developed metabolic labeling system to study endogenous protein complexes.

Two other papers report new methods based on protein-fragment complementation, in which interaction between two proteins fused to complementary fragments of a reporter protein results in functional complementation and reporter activity. Although not yet implemented in high-throughput format, these types of techniques allow the observation of protein interaction in living cells, some with subcellular resolution. On page 977, the original developer of the protein-fragment complementation assay, Stephen Michnick, describes an improved assay with better sensitivity and the capacity to measure reversible interactions. As an alternative, Moritz Rossner and colleagues (p. 985) describe the first splitprotease assay, which exploits a protease to activate secondary reporters of choice, thus providing greater flexibility in the readout and expanding the possibilities for multiplexing.
An additional perk of complementation assays is that native protein localization can usually be maintained, provided that expression levels are kept low. Explanation of some protein behaviors, however, will require observation of interaction events between native proteins in their natural environment. This, of course, is a challenge of both sensitivity and specificity. But one that was met, at least in fixed cells, by the group of Ulf Landegren who adapted the proximity ligation assay to allow interaction detection in situ (p. 995).

Ultimately, single-molecule studies may be necessary to obtain complete descriptions of some protein interactions. Atomic force microscopy (AFM), for example, can quantify interaction forces between individual proteins. In this issue Simon Scheuring's team (p. 1007), describing the first AFM measurements on unsupported patches of membrane, shows how this technique may even help to settle many outstanding questions regarding membrane-protein organization and interactions.

Transmembrane proteins, in fact, constitute a difficult case, for which none of the common techniques of interaction measurement are particularly well adapted. Their membrane dependence complicates many assays, and the associated spatial constraints potentially result in confounding random interactions. The case of the G protein-coupled receptor (GPCR) family discussed on page 1001 illustrates this situation. Based on recent evidence, many researchers now accept that GPCRs exist primarily as functional dimers. However, Simon Davis' group examines the question of receptor dimerization using a more quantitative framework to distinguish true dimerization from random interactions. Their results question the notion that many of these receptors dimerize.

This example illustrates how different methods, owing to inherent biases or limitations, may provide different answers to interaction questions. It is therefore crucial to multiply the approaches, varying the window through which one looks at an interaction, to validate observations and get past the limitations of each technique. With this in mind, we hope you enjoy this array of new methods, and the accompanying News and Views that compare them and put them into perspective.

\title{
Call for comments!
}

\section{Nature Methods is pleased to introduce methagora, an online space to debate methodological affairs.}

Methodological questions, big and small, are at the core of everyone's research, day after day, technology after technology. Now and again, a methodological question even affects an entire field of research-for example, when divergent methodological approaches provide contradicting results or when the misuse of a method in a specific context risks casting doubt on its reliability in general.

We believe that both seasoned users and neophytes-as well as editors-can learn a lot from constructive discussions about these issues. Sometimes a simple clarification from experts will suffice, other times multiple opinions will have to be heard and weighed for a better understanding of the methodological problems at stake.

To facilitate such discussions, Nature Methods is pleased to introduce methagora, an online commenting forum dedicated to methodological topics.

Regularly, the editors will seed a debate by posting a topic and inviting comments. Most often, the topic will be directly linked to a paper recently published in the journal, but we will also consider unrelated methodological issues of importance to a particular community of scientists. And then, we call for your participation!

Commenting is simple. Comments submitted online will be posted after a rapid screen by the editors to ensure that they are relevant, appropriate and free of obvious commercial interests. We encourage, but do not require, authors to sign their posted comments, and we request a valid email address only to allow correspondence between the author of the comment and the editors.

Currently methagora is hosting a discussion on the most appropriate way of minimizing the reporting of false positives in large-scale RNAi screens. We are seeking opinions from a cross-section of the community of RNAi users in the hope of reaching a consensus of best practices that can be used as reference for users and editors.

The second ongoing debate concerns methods to measure proteinprotein interactions in the particular case of $G$ protein-coupled receptors (GPCRs). The results presented by Davis and colleagues in this issue (p. 1001), which call into question the widely accepted notion that all GPCRs exist as functional dimers, invite reflections on the native form of these proteins, but also on the methods used to determine their interactions.

We trust you will be interested in these debates and in forthcoming topics. So please visit the methagora site frequently and participate in the discussions. Have your say on the methods at the core of your research!

Methagora can be found at http://blogs.nature.com/nmeth/ methagora/ 